RASĀYAN J. Chem.

Vol. 13 | No. 1 |291 - 297| January - March | 2020 ISSN: 0974-1496 | e-ISSN: 0976-0083 | CODEN: RJCABP

\title{
SYNTHESIS OF FEW 1,3,4-OXADIAZOLE DERIVATIVES BLENDED WITH DIFFERENT HETEROCYCLES AND THEIR IN-VITRO ANTIBACTERIAL ACTIVITIES
}

\author{
M. Idrees ${ }^{1, *}$, Y.G. Bodkhe ${ }^{2}$, N. J. Siddiqui ${ }^{1}$ and S. Kola ${ }^{2}$ \\ ${ }^{1}$ Department of Chemistry, Institute of Science, Nagpur-440001 (Maharashtra), India \\ ${ }^{2}$ Department of Chemistry, Government Science College, \\ Gadchiroli-442605 (Maharashtra), India \\ *E-mail: idreesshaikh.2009@gmail.com
}

\begin{abstract}
In the present article, we have described the synthesis of few novel 1,3,4-oxadiazole derivatives (4a-f) blended with quinoline, benzofuran and pyrazole nuclei obtained by the cyclization followed by acetylation of corresponding carbohydrazides (3a-f) with acetic anhydride. Intermediary compounds (3a-f) were obtained by condensation of 5(benzofuran-2-yl)-1-phenyl-1 $H$-pyrazole-3-carbohydrazide (1) with 2-( $p$-tolyloxy) substituted quinoline-3carbaldehydes (2a-f) in the presence of the small amount of acetic acid in ethanol. The structure of synthesized compounds was confirmed through spectral studies like IR, ${ }^{1} \mathrm{HNMR},{ }^{13} \mathrm{CNMR}$ and Mass spectra's besides elemental analysis. All the synthesized compounds were assessed for in-vitro antibacterial activities against $S$. aureus and $E$. coli, the consequence of assessment was compared with as standard reference drug.
\end{abstract}

Keywords: 1,3,4-Oxadiazole, Quinoline, Benzofuran, Pyrazole, $p$-Tolyloxy, Carbohydrazide

(C) RASĀYAN. All rights reserved

\section{INTRODUCTION}

Heterocyclic molecules hold a core stage in the development of molecules to enhance the quality of human life. Perhaps more than seventy percent of the drugs used today are heterocyclic compounds. Literature survey on heterocycles containing oxygen and nitrogen indicated that oxadiazoles are one of such heterocycles utilized in the synthesis of commercial drugs and are easily accessible in the market like Furamizole as an antibiotic, Tiodazosin ${ }^{1}$ and Nesapidil ${ }^{2}$ as anti-hypertensive agents. Among different isomers, 1,3,4-oxadiazole is a prolific source of bioactivity in the area of drug discovery and its derivatives had been reported to exhibit abundant activities like antiviral ${ }^{3}$, anticancer ${ }^{4-5}$, antibacterial ${ }^{6-10}$, insecticidal $^{11}$, anti-allergic ${ }^{12}$, HIV integrated inhibitors ${ }^{13}$, anti-tubercular ${ }^{14-16}$, anticonvulsant ${ }^{17}$, tubulin inhibitors $^{18}$, analgesic ${ }^{19}$, antitumor ${ }^{20}$, anti-inflammatory ${ }^{21-23}$ and many more biological activities. ${ }^{24-28}$ They are also useful as, lipoxygenase inhibitors ${ }^{29}$, and used as dye pigments. ${ }^{30}$

Several approaches have been existed in the literature to obtain oxadiazoles. Most frequently 1,3,4oxadiazoles are obtained by reactions of hydrazine or acid hydrazides with acid chlorides or carboxylic acids and direct cyclization of diacylhydrazines utilizing different dehydrating agents. Thus, an extensive literature survey on this heterocycle prompted us to continue our research by designing and synthesizing few novel 1,3,4-oxadiazole derivatives blending them with quinoline, benzofuran and pyrazole rings with a view that inclusion of all these moieties in a single molecule may enhance the biological profile of ultimate compound to a greater extent. Consecutively, it was also planned to carry out the in-vitro antibacterial assessment of these synthesized compounds.

\section{Material and Methods}

\section{EXPERIMENTAL}

AR grade chemicals of Merck and Sigma Aldrich are used for the synthesis. E. Merck TLC aluminium sheet was used to monitor the reactions and UV cabinet was used for visualization of spot. Melting points Rasayan J. Chem., 13(1), 291-297(2020) http://dx.doi.org/10.31788/RJC.2020.1315593 
were noted in the open capillary in the paraffin bath and are uncorrected. Bruker AM 400 instruments were used to record ${ }^{1} \mathrm{HNMR}$ and ${ }^{13} \mathrm{C}$ NMR spectra in DMSO- $d_{6}$ and $\mathrm{CDCl}_{3}$ as a solvent. Shimadzu IR Spectrophotometer was used to record IR spectra. ESI-MS mass spectra were noted in Waters Micromass Micro, Mass Spectrophotometer. Thermo Scientific Flash-2000 was used for Elemental analysis.

Synthesis of 5-(benzofuran-2-yl)- $N^{\prime}-(2-(p-t o l y l o x y)$ substituted quinolin-3-yl) methylene)-1-phenyl$1 H$-pyrazole-3-carbohydrazide ${ }^{31}$ (3a-f)

In a $100 \mathrm{~mL}$ R.B. flask, mixture of compound $(2,0.01 \mathrm{~mol})$ and carbohydrazide $(1,0.01 \mathrm{~mol})$ and $2-3$ drops of acetic acid were dissolved in ethanol then reaction content was refluxed for $2 \mathrm{~h}$. Resultant mass was added to crushed ice, filtered and recrystallized using 1,4-dioxane.

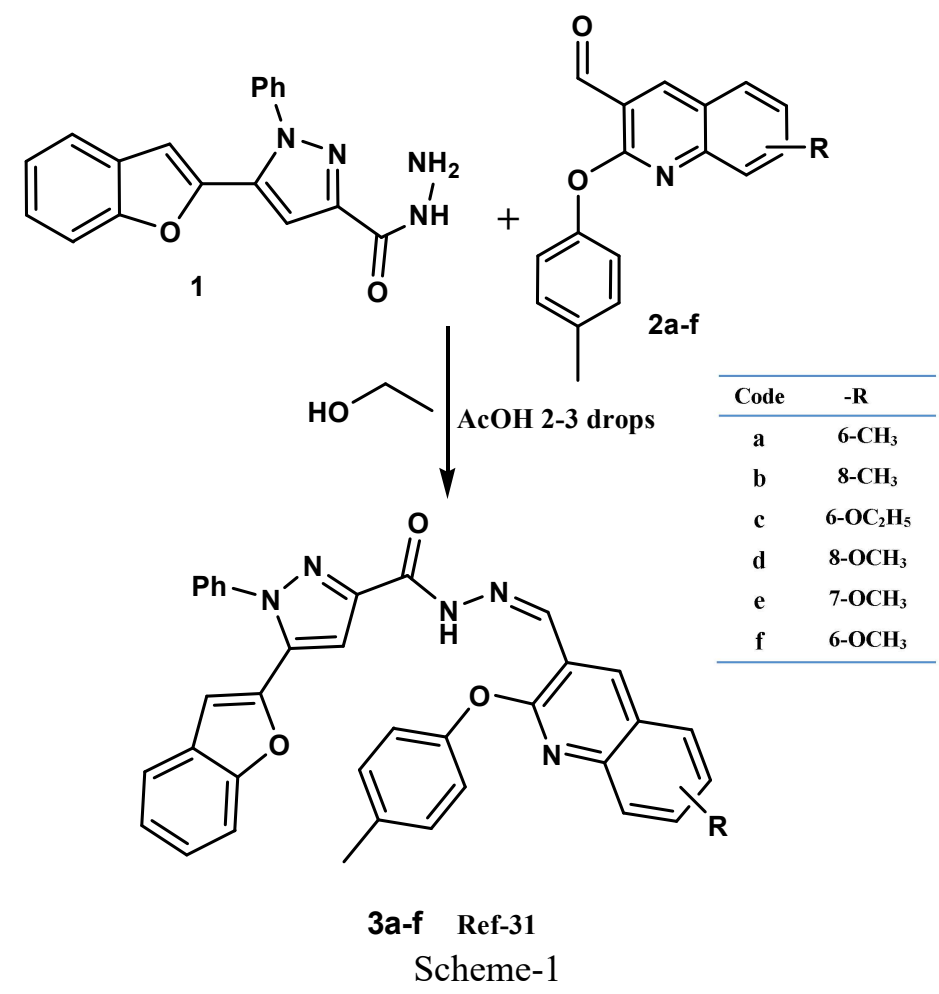

5-(Benzofuran-2-yl)- $N^{\prime}-((6-m e t h y l-2-(p-t o l y l o x y)-q u i n o l i n-3-y l) m e t h y l e n e)-1-p h e n y l-1 H$-pyrazole-3carbohydrazide (3a)

Yellow crystalline solid; m.p. $263-265^{\circ} \mathrm{C}$;yield: 81\%; IR (KBr, $\left.v_{\max }, \mathrm{cm}^{-1}\right)$ : 3062,3029 (C-H arom.), 2857,2915 (C-H sym., aliphatic), $1688(\mathrm{C}=\mathrm{O}), 1650-1600(\mathrm{C}=\mathrm{N}), 2958$ (C-H asym. aliph.), 1240 (C-O-C sym. ether), 1058 (C-O-C asym. ether), 3293, 3412(N-H); ${ }^{1} \mathrm{H}$ NMR (DMSO-d6) $\delta$ ppm: 2.47 (s, 3H, $\mathrm{CH}_{3}$ attached to quinoline ring), $2.36\left(\mathrm{~s}, 3 \mathrm{H}, \mathrm{Ar}-\mathrm{CH}_{3}\right), 12.21(\mathrm{~s}, 1 \mathrm{H}, \mathrm{NHCO}), 7.14-9.08(\mathrm{~m}, 19 \mathrm{H}$, arom.+ Heterocycl. ring protons).6.51 (s, $1 \mathrm{H}$, at C4of pyrazole ring); MS: m/e $578[\mathrm{M}+\mathrm{H}]^{+}, 577 \mathrm{M}^{+}, 579[\mathrm{M}+$ $2]^{+}, 600[\mathrm{M}+\mathrm{Na}]^{+}$. Elemental Analysis: for $\mathrm{C}_{36} \mathrm{H}_{27} \mathrm{~N}_{5} \mathrm{O}_{3}$; Calculated: $\mathrm{C}, 74.85 ; \mathrm{H}, 4.71 ; \mathrm{N}, 12.12$; Found: C, $74.75 ; \mathrm{H}, 4.68 ; \mathrm{N}, 12.10$

Similarly, spectral, physical and analytical data of $\mathbf{3 b}$-f $\mathbf{f}$ were also determined.

General Procedure for Synthesis of 1-(5-(5-(benzofuran-2-yl)-1-phenyl-1H-pyrazol-3-yl)-2-(2-(ptolyloxy) substituted quinolin-3-yl)-1, 3, 4-oxadiazol-3(2H)-yl) ethanone (4a-f)

3a $(0.001 \mathrm{~mol})$ and acetic anhydride $(10-15 \mathrm{~mL})$ was taken in a round bottom flask and refluxed for $5 \mathrm{~h}$. The resultant product obtained was added to crushed ice in a beaker and left undisturbed overnight, it was then filtered, washed, dried and recrystallized by 1,4-dioxane to obtain 4a-f derivatives. Similarly, $\mathbf{4 b - f}$ was obtained by using the same method as used for synthesizing $\mathbf{4 a}$. 


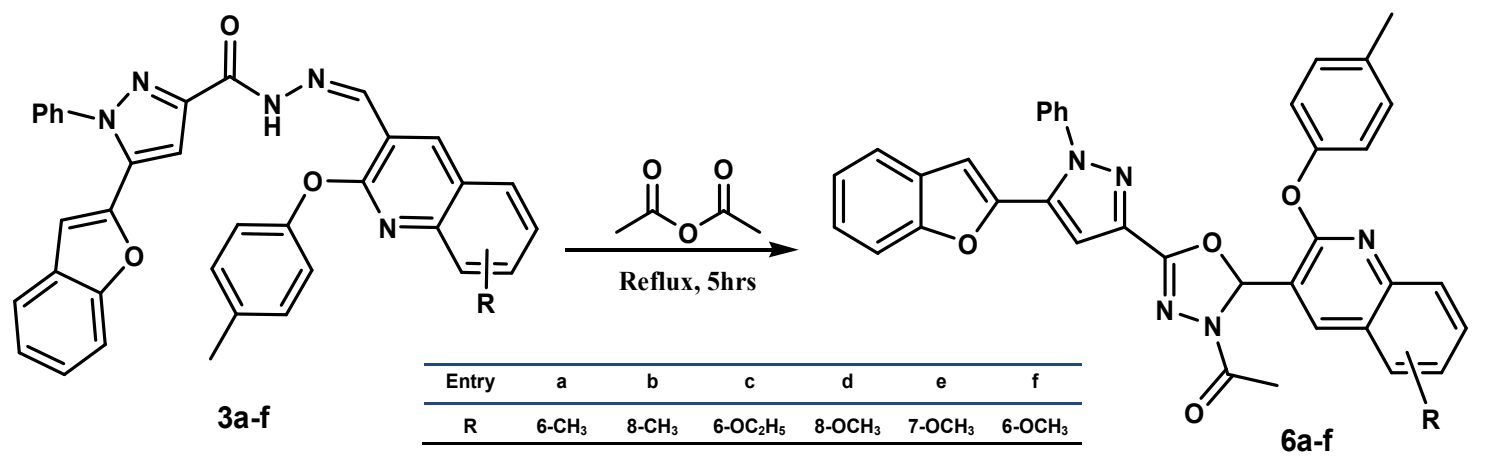

Scheme-2

1-(5-(5-(Benzofuran-2-yl)-1-phenyl-1H-pyrazol-3-yl)-2-(6-ethoxy-2-(p-tolyloxy) quinolin-3 -yl)-1,3,4oxadiazol-3(2H)-yl)ethanone $(4 \mathrm{c})$

Pale yellow crystalline solid; Recrystallizing solvent, 1,4-Dioxane; m.pt., 233-235 $\mathrm{C}$; Rf., 0.48; Yield, 78\%; M.F, $\mathrm{C}_{39} \mathrm{H}_{31} \mathrm{~N}_{5} \mathrm{O}_{5}$ IR: ( $\mathrm{KBr} v_{\max }$ in $\left.\mathrm{cm}^{-1}\right)$ : 3064(C-H arom.), 2980, 2930 (C-H asym. aliph.), 2880(C-H sym. aliph.), 1666 (C=O amide.), $1621(\mathrm{C}=\mathrm{N}$ pyrazole.), 1225,1258(C-O-C asym. pyrazole moiety), 1595(C=C quinoline.), 1444,1467(C-H asym. def., aliph.), 1395 (C-H sym. def., aliph.), 1000,1051 (C-H. i.p.def., arom.), 827(C-H. o.o.p.def., arom.). 1051 (C-O-C sym. oxadiazole moiety), 1051 (N-N oxadiazole). ${ }^{1} \mathrm{H}$ NMR (DMSO-d 6$): \delta(\mathrm{ppm})$ 1.41-1.45(t, $\left.3 \mathrm{H}, \mathrm{Ar}-\mathrm{OCH}_{2} \underline{\mathrm{CH}}_{3}\right), 2.32\left(\mathrm{~s}, 3 \mathrm{H}, \mathrm{CH}_{3}\right.$ group attached to $-\mathrm{COCH}_{3}$ group), 2.39 (s, $3 \mathrm{H}, \mathrm{CH}_{3}$ group attached to phenyl ring), 4.16-4.17(q, $2 \mathrm{H}, \mathrm{Ar}-$ $\left.\mathrm{OCH}_{2} \mathrm{CH}_{3}\right), 6.47\left(\mathrm{~s}, 1 \mathrm{H}\right.$, one proton at $\mathrm{C}_{4}$ of pyrazole ring, $8.42\left(\mathrm{~s}, 1 \mathrm{H}\right.$, one proton at $\mathrm{C}_{4}$ of quinoline ring), 7.18-7.89 $\left(\mathrm{m}, 18 \mathrm{H}\right.$, aromatic and hetero aromatic ring protons). ESI-MS $(\mathrm{m} / \mathrm{z}): 650[\mathrm{M}+\mathrm{H}]^{+}$. Elemental Analysis: for $\mathrm{C}_{39} \mathrm{H}_{31} \mathrm{~N}_{5} \mathrm{O}_{5}$; Calculated: $\mathrm{C}, 72.10 ; \mathrm{H}, 4.81 ; \mathrm{N}, 10.78$; Found: $\mathrm{C}, 72.14 ; \mathrm{H}, 4.78 ; \mathrm{N}, 10.80$.

\section{1-(2-(2-(p-Tolyloxy)-6-methylquinolin-3-yl)-5-(5-(benzofuran-2-yl)-1-phenyl-1H-pyrazole-3-yl)-} 1,3,4-oxadiazol-3(2H)-yl)ethanone (4a)

IR: $\left(\mathrm{KBr} v_{\max }\right.$ in $\left.\mathrm{cm}^{-1}\right): 3065(\mathrm{C}-\mathrm{H}$ arom. $), 1664(\mathrm{C}=\mathrm{O}$ amide. $), 1624(\mathrm{C}=\mathrm{N}$ pyrazole. $), 1597(\mathrm{C}=\mathrm{C}$ quinoline.), 1446,1465(C-H asym. def., aliph.), 1398(C-H sym. def., aliph.), 1001,1053(C-H. i.p.def., arom.), 825(C-H. o.o.p.def., arom.). 1053(C-O-C sym. oxadiazole moiety), 1222,1251(C-O-C asym. pyrazole moiety), 1053(N-N oxadiazole).

\section{1-(2-(2-(p-Tolyloxy)-8-methylquinolin-3-yl)-5-(5-(benzofuran-2-yl)-1-phenyl-1H-pyrazole-3-yl)-} 1,3,4-oxadiazol-3(2H)-yl)ethanone(4b)

IR: $\left(\mathrm{KBr} v_{\max }\right.$ in $\left.\mathrm{cm}^{-1}\right): 3070(\mathrm{C}-\mathrm{H}$ arom. $), 2884(\mathrm{C}-\mathrm{H}$ sym. aliph. $), 1663(\mathrm{C}=\mathrm{O}$ amide. $), 1620(\mathrm{C}=\mathrm{N}$ pyrazole.), 1592(C=C quinoline.), 1445,1469(C-H asym. def., aliph.), 1391(C-H sym. def., aliph.), 1005,1053(C-H. i.p.def., arom.), 829(C-H. o.o.p.def., arom.). 1056(C-O-C sym. oxadiazole moiety), 1227,1255 (C-O-C asym. pyrazole moiety), 1053(N-N oxadiazole).

\section{1-(2-(2-(p-Tolyloxy)-8-methoxyquinolin-3-yl)-5-(5-(benzofuran-2-yl)-1-phenyl-1H-pyrazol-3-yl)-} 1,3,4-oxadiazol-3(2H)-yl)ethanone(4d)

IR: $\left(\mathrm{KBr} v_{\max }\right.$ in $\left.\mathrm{cm}^{-1}\right)$ : 3066(C-H arom. $), 2882(\mathrm{C}-\mathrm{H}$ sym. aliph. $), 1662(\mathrm{C}=\mathrm{O}$ amide. $), 1623(\mathrm{C}=\mathrm{N}$ pyrazole.), 1596(C=C quinoline.), 1442,1462(C-H asym. def., aliph.), 1396(C-H sym. def., aliph.), 1002,1054(C-H. i.p.def., arom.), 829(C-H. o.o.p.def., arom.). 1056(C-O-C sym. oxadiazole moiety), $1226,1260(\mathrm{C}-\mathrm{O}-\mathrm{C}$ asym. pyrazole moiety), 1053(N-N oxadiazole).

1-(2-(2-(p-Tolyloxy)-7-methoxyquinolin-3-yl)-5-(5-(benzofuran-2-yl)-1-phenyl-1H-pyrazole-3-yl)1,3,4-oxadiazol-3(2H)-yl)ethanone $(4 \mathrm{e})$

IR: $\left(\mathrm{KBr} v_{\max }\right.$ in $\left.\mathrm{cm}^{-1}\right)$ : 3067(C-H arom. $), 2882(\mathrm{C}-\mathrm{H}$ sym. aliph. $), 1668(\mathrm{C}=\mathrm{O}$ amide. $), 1623(\mathrm{C}=\mathrm{N}$ pyrazole.), 1597(C=C quinoline.), 1448,1470(C-H asym. def., aliph.), 1399(C-H sym. def., aliph.), 
RASĀYAN J. Chem.

Vol. 13 | No. 1 | 291 - 297| January - March | 2020

1001,1058(C-H. i.p.def., arom.), 825(C-H. o.o.p.def., arom.). 1057(C-O-C sym. oxadiazole moiety), 1229,1262(C-O-C asym. pyrazole moiety), 1045(N-N oxadiazole).

\section{1-(2-(2-(p-Tolyloxy)-6-methoxyquinolin-3-yl)-5-(5-(benzofuran-2-yl)-1-phenyl-1H-pyrazole-3-yl)- 1,3,4-oxadiazol-3(2H)-yl)ethanone (4f)}

IR: $\left(\mathrm{KBr} v_{\max }\right.$ in $\left.\mathrm{cm}^{-1}\right)$ : 3071(C-H arom.), 2985, 2883(C-H sym. aliph.), 1669(C=O amide.), 1625(C=N pyrazole.), 1598( $\mathrm{C}=\mathrm{C}$ quinoline.), 1446,1469(C-H asym. def., aliph.), 1399(C-H sym. def., aliph.), 1005,1049(C-H. i.p.def., arom.), 825(C-H. o.o.p.def., arom.). 1046(C-O-C sym. oxadiazole moiety), 1228,1265(C-O-C asym. pyrazole moiety), 1057(N-N oxadiazole).

Table-1: Physical and Analytical Data of Substituted 1,3,4-Oxadiazoles (4a-f)

\begin{tabular}{|c|c|c|c|c|c|}
\hline \multirow[t]{2}{*}{ Entry } & \multirow{2}{*}{$\begin{array}{c}\text { 1-(5-(5-(benzofuran-2-yl)-1- } \\
\text { phenyl-1H-pyrazol-3-yl)-2-(2-( } p \text { - } \\
\text { tolyloxy) substituted quinolin-3- } \\
\text { yl)-1, 3, 4-oxadiazol-3(2H)-yl) } \\
\text { ethanone }(4 \mathrm{a}-\mathrm{f})\end{array}$} & \multirow{2}{*}{\multicolumn{3}{|c|}{ Physical Data (4a-f) }} & $\begin{array}{c}\text { Elemental Analysis } \\
\% \text { of } \mathrm{N}\end{array}$ \\
\hline & & & & & (Calcd) \\
\hline \multirow{6}{*}{$4 a$} & & M.F. & $:$ & $\mathrm{C}_{38} \mathrm{H}_{29} \mathrm{~N}_{5} \mathrm{O}_{4}$ & \multirow{3}{*}{$\mathrm{N}, 11.23$} \\
\hline & & Colour & $:$ & Yellow & \\
\hline & & M.Pt & $:$ & $160-164^{\circ} \mathrm{C}$ & \\
\hline & & Yield & $:$ & $80 \%$ & \multirow{3}{*}{$(\mathrm{N}, 11.30)$} \\
\hline & & Rf. & $:$ & 0.62 & \\
\hline & & Recys. S & $:$ & 1, 4 dioxane & \\
\hline \multirow{6}{*}{$4 b$} & & M.F & $:$ & $\mathrm{C}_{38} \mathrm{H}_{29} \mathrm{~N}_{5} \mathrm{O}_{4}$ & \multirow{3}{*}{$\mathrm{N}, 11.12$} \\
\hline & & Colour & $:$ & Yellow & \\
\hline & & M.Pt & $:$ & $220-218^{\circ} \mathrm{C}$ & \\
\hline & & Yield & $:$ & $80 \%$ & \multirow{3}{*}{$(\mathrm{N}, 11.30)$} \\
\hline & & Rf. & $:$ & 0.44 & \\
\hline & & Recys. S & $:$ & 1, 4 dioxane & \\
\hline \multirow{6}{*}{$4 d$} & & M.F & $:$ & $\mathrm{C}_{38} \mathrm{H}_{29} \mathrm{~N}_{5} \mathrm{O}_{5}$ & \multirow{3}{*}{$\mathrm{N}, 11.42$} \\
\hline & & Colour & $:$ & Yellow & \\
\hline & & M.Pt & $:$ & $214-216^{\circ} \mathrm{C}$ & \\
\hline & & Yield & $:$ & $80 \%$ & \multirow{3}{*}{$(\mathrm{N}, 11.02)$} \\
\hline & & Rf. & $:$ & 0.52 & \\
\hline & & Recys. S & $:$ & 1, 4 dioxane & \\
\hline \multirow{6}{*}{$4 \mathrm{e}$} & & M.F & $:$ & $\mathrm{C}_{38} \mathrm{H}_{29} \mathrm{~N}_{5} \mathrm{O}_{5}$ & \multirow{3}{*}{$\mathrm{N}, 11.02$} \\
\hline & & Colour & $:$ & Yellow & \\
\hline & & M.Pt & $:$ & $182-184^{\circ} \mathrm{C}$ & \\
\hline & & Yield & $:$ & $79 \%$ & \multirow{3}{*}{$(\mathrm{N}, 11.02)$} \\
\hline & & Rf. & $:$ & 0.35 & \\
\hline & & Recys. S & $:$ & 1,4 dioxane & \\
\hline \multirow{6}{*}{$4 f$} & & M.F & $:$ & $\mathrm{C}_{38} \mathrm{H}_{29} \mathrm{~N}_{5} \mathrm{O}_{5}$ & \multirow{3}{*}{$\mathrm{N}, 11.08$} \\
\hline & & Colour & $:$ & Yellow & \\
\hline & & M.Pt & $:$ & $197-199^{\circ} \mathrm{C}$ & \\
\hline & & Yield & $:$ & $82 \%$ & \multirow{3}{*}{$(\mathrm{N}, 11.02)$} \\
\hline & & Rf. & $:$ & 0.57 & \\
\hline & & Recys. S & $:$ & 1, 4 dioxane & \\
\hline
\end{tabular}

\section{Antibacterial Activity}

Test solution of each compound (4a-f) was organized by dissolving calculated weight compound in DMSO and diluted appropriately to give the subsequent strength of $31-1000 \mu \mathrm{g} / \mathrm{mL}$. Nutrient Broth Medium and Nutrient Agar Media, and all plates were sterilized in an autoclave at room temperature for 30 minutes. Microorganism strains were kept at $37^{\circ} \mathrm{C}$ in nutrient agar medium. Petri plates were ready by spreading $10 \mathrm{~mL}$ of Nutrient Agar Media for bacteria. Diluted bacterial suspension of $0.1 \mathrm{~mL}$ of culture was banquet over Nutrient Agar Media in plate. Sterile paper discs Whatmann No.1 (6mm) were soaked 
RASĀYAN J. Chem.

Vol. 13 | No. 1 | 291 - 297| January - March | 2020

with a solution and dried at room temperature. Soaked discs of test solution were applied and the plates were incubated for $24 \mathrm{~h}$ (bacteria) at $37^{\circ} \mathrm{C}$ and the zone of inhibition was measured in four directions in $\mathrm{mm}$ and expressed as mean.

\section{RESULTS AND DISCUSSION}

All of the 1,3,4-oxadiazole derivatives 4a-f blended with different heterocycles were synthesized fruitfully, with the ensuing aim of finding novel compounds with potential antibacterial activities. The synthetic protocol has been outlined in reaction scheme 2 . Structures of all obtained compounds $\mathbf{4 a - f}$ has been characterized by spectral data such as FT-IR, ${ }^{1} \mathrm{HNMR},{ }^{13} \mathrm{CNMR}$ and mass spectra and elemental analysis. At each step the reaction was observed with TLC, solubility and melting point were determined for all the final and intermediate products. The synthesis of target compounds 1,3,4-oxadiazoles (4a-f) was carried out by cyclisation cum acetylation of intermediate 3-carbohydrazide (3a-f) derivatives on reaction with acetic anhydride. IR spectra of compound $\mathbf{4 c}$ shows the absence of absorption band in the range $3393,3147 \mathrm{~cm}^{-1}$ due to $-\mathrm{NH}$ group which were found to be present in the $\mathbf{3 c}$ but characteristic absorption bands were observed at 1051 and $1258 \mathrm{~cm}^{-1}$ due to C-O-C symmetric and asymmetric stretch respectively of oxadiazole moiety which confirms the formation of oxadiazole ring. Another evidence was obtained from ${ }^{1} \mathrm{HNMR}$ for $4 \mathbf{c}$ that exhibited two singlet's, one appeared at $\delta 2.32 \mathrm{ppm}$ and other at $\delta 2.39 \mathrm{ppm}$ due to three protons of each $-\mathrm{CH}_{3}$ group attached to carbonyl group and aromatic ring respectively. Triplet in the range of $\delta 1.41-1.45 \mathrm{ppm}$ appeared due to three protons of $-\mathrm{CH}_{3}$ in $\mathrm{Ar}-$ $\mathrm{OCH}_{2} \mathrm{CH}_{3}$ and a quartet in between $\delta$ 4.16-4.17 ppm for two protons indicating that $-\mathrm{CH}_{2}$ is attached to $\mathrm{CH}_{3}$ and phenoxy group in $\mathrm{Ar}-\mathrm{OCH}_{2} \mathrm{CH}_{3}$, multiplet signals for eighteen protons due to aromatic and hetero aromatic ring appeared in their expected region from $\delta 7.18-7.89 \mathrm{ppm}$. One singlet was observed at $\delta 6.47 \mathrm{ppm}$ due to one proton at $\mathrm{C}_{4}$ of pyrazole ring, and another singlet at $\delta 8.42 \mathrm{ppm}$ due one proton of $\mathrm{C}_{4}$ of quinoline ring. Absence of a signal for $-\mathrm{NH}$ proton as expected in the reactant confirms that cyclization has occurred to get target molecule $4 \mathbf{c}$. One more evidence for the formation $4 \mathbf{c}$ came from its mass spectra which exhibited the molecular ion peak $\mathrm{m} / z$ at $650[\mathrm{M}+\mathrm{H}]^{+}$. From all the above spectral and analytical analysis the molecular weight and formula of $4 \mathbf{c}$ is found to be 649 and $\mathrm{C}_{39} \mathrm{H}_{31} \mathrm{~N}_{5} \mathrm{O}_{5}$ respectively. Similarly, elemental analysis result of compound shows carbon, hydrogen and nitrogen as $72.14,4.78$ and 10.80 percent respectively.

Table-2: Antibacterial Activities of 4a-f

\begin{tabular}{|c|c|c|c|c|c|c|c|c|c|c|c|c|}
\hline \multicolumn{13}{|c|}{ Zone of Inhibition (mm) } \\
\hline \multirow{4}{*}{$\begin{array}{l}\text { Compd. } \\
\text { Code }\end{array}$} & \multicolumn{6}{|c|}{ Gram +ve } & \multicolumn{6}{|c|}{ Gram -ve } \\
\hline & \multicolumn{6}{|c|}{ S. aureus } & \multicolumn{6}{|c|}{ E. coli } \\
\hline & \multicolumn{6}{|c|}{ Conc. $(\mu \mathrm{g} / \mathrm{mL})$} & \multicolumn{6}{|c|}{ Conc. $(\mu \mathrm{g} / \mathrm{mL})$} \\
\hline & 1000 & 500 & 250 & 125 & 63.5 & 31 & 1000 & 500 & 250 & 125 & 63.5 & 31 \\
\hline $4 a$ & 22 & 20 & 21 & 17 & 15 & 13 & 23 & 25 & 20 & 20 & 15 & 13 \\
\hline $4 \mathrm{~b}$ & 23 & 21 & 20 & 18 & 19 & 16 & 21 & 22 & 19 & 16 & 17 & 15 \\
\hline $4 \mathrm{c}$ & 17 & 19 & 17 & 14 & 12 & 11 & 20 & 20 & 23 & 22 & 14 & 11 \\
\hline $4 \mathrm{~d}$ & 24 & 20 & 20 & 1 & 18 & 12 & 26 & 22 & 19 & 17 & 16 & 13 \\
\hline $4 \mathrm{e}$ & 20 & 18 & 16 & 15 & 12 & 13 & 24 & 21 & 18 & 16 & 15 & 12 \\
\hline $4 \mathrm{f}$ & 18 & 19 & 18 & 17 & 15 & 10 & 16 & 19 & 16 & 14 & 11 & 10 \\
\hline DMSO & - & - & - & - & - & - & - & - & - & - & - & - \\
\hline Std. Drug & 24 & 22 & 20 & 19 & 17 & 15 & 26 & 24 & 23 & 21 & 17 & 14 \\
\hline
\end{tabular}

\section{Antibacterial Activity}

The entire innovative heterocyclic compounds 4a-f was assessed for their in-vitro antibacterial activity. Results acquired are summarized in the Table-2. From the bioassay outcome it is revealed that the compounds showed variable activity against different bacteria. This dissimilarity in activity can be recognized due to the modification of structures and functional groups attached to the prominent nucleus 1,3,4-oxadiazole which associated with a huge biological perspective or may be by virtue of toxophoric quinoline and benzofuran nuclei.

\section{CONCLUSION}

In summary, we have synthesized six novel 1,3,4-oxadiazole 4a-f derivatives in excellent yields. The structure of newly afforded compounds was recognized by spectroscopic study and chemical 
RASĀYAN J. Chem.

Vol. 13 | No. 1 | 291 - 297| January - March | 2020

investigation. Among the synthesized compounds all the compounds show good to reasonable activity with selected bacteria $S$. aureus and $E$. coli at various concentrations.

\section{ACKNOWLEDGMENT}

The writers are gratified to Dr. Roshan Nasare for support and cooperation during the entire research. The authors are also thankful to the Head of research centre Govt. Science College, Gadchiroli for permitting research laboratory. Similarly, the authors are also obliged to the Director, SAIF, Punjab University, Chandigarh for providing CHN analysis, IR, ${ }^{1} \mathrm{HNMR}$, Mass and ${ }^{13} \mathrm{CNMR}$ Spectra.

\section{REFERENCES}

1. M. Ogata, H. Atobe, H. Kushida, K. Yamamoto, Journal of Antibiotics, 24(7), 443(1971), DOI: 10.7164/ antibiotics.24.443.

2. R. Schlecker, P.C. Thieme,Tetrahedron, 44(11), 3289(1988), DOI: 10.1016/S0040-4020(01)85962-7.

3. Z. Li, P. Zhan, X. Liu, Mini Reviews in Medicinal Chemistry, 11(13), 1130(2011), DOI: $10.2174 / 138955711797655407$.

4. M. Abdel-Aziz, K.A. Metwally, M.A. Gamal-Eldeen, M.O. Aly, Anti-Cancer Agents in Medicinal Chemistry, 16, 269 (2016), DOI:10.2174 /1871520 615666150907093855.

5. R.S. Subrahmanyam, P. Ramesh, B.S. Krishna, D.S. Swaroop, M.A. Khan, M.M. Darla, K. Adeppa, B.V. Bhaskar, W. Rajendra and A. Venkateswara Rao, Rasayan Journal of Chemistry, 10(4), 1194 (2017), DOI:10. 7324/RJC.2017.1041838.

6. A. Rehman, A. Siddiqa, M.A. Abbasi, S. Rasool, S. Siddiqui, I. Ahmad, S. Afzal, Bulletin of Faculty of Pharmacy, 53(1), 37 (2015), DOI:10.1016/j.bfopcu.2014.10.001.

7. S. Bhati, Rasayan Journal of Chemistry, 11(3), 1366 (2018), DOI:10.31788/RJC.2018.1132066.

8. S. Ningaiah, U.K. Bhadraiah, S.D. Doddaramappa, S. Keshavamurthy, C. Javarasetty, Bioorganic and Medicinal Chemistry Letters, 24(1), 245(2014), DOI:10.1016/j.bmcl.2013.11.029.

9. K. Ramalingam, D. Ramesh, B. Sreenivasulu, Rasayan Journal of Chemistry, 12(2), 583(2019), DOI: 10.31788/RJC.2019.1225096.

10. G. Vijaya Bhasker, E. Laxminarayana, A. Latha and M. Thirumala Chary, Rasayan Journal of Chemistry, 10(4), 1521(2017), DOI: 10.7324/RJC.2017.1041920.

11. T.P. Mohan, B. Vishalakshi, K.S. Bhat, G.N. Kendappa, Indian Journal of Chemistry, 43B, 1798 (2004).

12. D. Reddy Guda, S.J. Park, Lee, European Journal of Medicinal Chemistry, 62, 84(2013), DOI:10.1016/j.ejmech.2012.12.035.

13. M. Akhter, A. Husain, B. Azad, A. Mohammad, European Journal of Medicinal Chemistry, 44(6), 2372 (2009), DOI:10.1016/j.ejm ech.2008.09.005.

14. P. P. Jain, M. S. Degani, A. Raju, A. Anantram, M. Seervi, S. Sathaye, M.Ray, M.G. Rajan, Bioorga nic and Medicinal Chemistry Letters, 26(2), 645 (2016), DOI:10.1016 /j.bmcl .2015 .11.057.

15. G.V.S. Kumar, Y. Rajendraprasad, B.P. Mallikarjuna, S.M. Chandrashekar, C. Kistayya, European Journal of Medicinal Chemistry, 45(5), 2063 (2010), DOI:10.1016/j. ejmech.2010.01.045.

16. P.R. Shashikant, P.A. Rabara, P.S. Jayashri, Indian Journal of Chemistry, 48B, 1453 (2009).

17. A. Ali, A. Sayyed, M.F. Kebriaeezadeh, D.N. Mehrabi, S. Abbas, Bioorganic and Medicinal Chemistry Letters, 14(24), 6057 (2004), DOI:10.1016/j.bmcl.2004.09.072.

18. A.G. Andrei, V. Andrey, M. Krasavin, T.L. Nguyen, E. Hamel, Bioorganic and Medicinal Chemistry Letters, 23(5), 1262 (2013), DOI:10.1016/j.bmcl.2013.01.007.

19. A. Shafiee, M. Abdollahi, A. Noeparast, N. Shahrokhinejad, N. Vousooghi, S.A. Tabatabai, R. Khorasani, Medicinal Chemistry Research, 20(4), 435 (2011), DOI: 10.1007/s00044-010-9335-0.

20. S. Bondock, S. Adel, H.A. Etman, F.A. Badria, European Journal of Medicinal Chemistry, 48, 192 (2012), DOI:10.10 16/j.ejmech.2011.12.013.

21. S.M. Sondhi, R. Rani, J. Singh, P. Roy, S.K. Agrawal and A.K. Saxena, Bioorganic and Medicinal Chemistry Letters, 20(7), 2306 (2010), DOI:10.1016 /j.bmcl.2010.01.147.

22. M. Akhter, A. Husain, B. Azad, A. Mohd., European Journal of Medicinal Chemistry, 44(6), 2372 (2009), DOI:10.1016/j.ejm ech.2008.09.005. 
RASĀYAN J. Chem.

Vol. 13 | No. 1 | 291 - 297| January - March | 2020

23. G. Nagalakshmi, Indian Journal of Pharmaceutical Sciences, 70(1), 49(2008), DOI:10.4103/0250474X.40331.

24. M. Ispikoudi, M. Amvrazis, C. Kontogiorgis, E.K. Alexandros, L. Konstantinos, D. HadjipavlouLitina, K.C. Fylaktakidou, European Journal of Medicinal Chemistry, 45(12), 5635(2010), DOI: 10.1016/j.ejmech.2010.09.016.

25. V.V. Dabholkar, R.P. Gavande, Rasayan Journal of Chemistry, 3(4), 655(2010).

26. S.G. Kucukguzel, E.E. Oruc, S. Rollas, F. Sahin, A. Ozbek, European Journal of Medicinal Chemistry, 37(3), 197(2002), DOI:10.1016/S0223-5234(01) 01326-5.

27. S. Bhati, Rasayan Journal of Chemistry, 11(3), 1366 (2018), DOI: 10.31788/RJC.2018.1132066.

28. B. Chandrakantha, P. Shetty, V. Nambiyar, N. Isloor, A.M. Isloor, European Journal of Medicinal Chemistry, 45(3), 1206 (2010), DOI:10.1016/j.ejmech.2009.11.046.

29. S. Rasool, Aziz-ur-Rehman, M. A. Abbasi, S. Z. Siddiqui, S.A.A. Shah, Brazilian Journal of Pharmaceutical Sciences, 52(3), 471 (2016), DOI:10.1590/s1984-82502016000300013.

30. X. Li, D. He, Dyes and Pigments, 93(1-3), 1422 (2012), DOI:10.1016/j.dyepig.2011.10.004.

31. M. Idrees, Y.G. Bodkhe, N.J. Siddiqui, Asian Journal of Chemistry, 30(10), 2361(2018), DOI: 10.14233/ajchem.2018.21522.

[RJC-5593/2019] 